\title{
New Perspectives and Competences in Redesign Clinical Organization and Appropriateness in Internal Medicine Wards: The Impact of ICT
}

\author{
Ilario Stefani* and Antonino Mazzone \\ Medicine Department, Azienda Socio Sanitaria Territoriale Ovest Milanese, Italy
}

*Corresponding author: Ilario Stefani, Medicine Department, Azienda Socio Sanitaria Territoriale Ovest Milanese, Legnano, Italy.

To Cite This Article: Ilario Stefani. New perspectives and competences in redesign clinical organization and appropriateness in Internal Medicine Wards: The impact of ICT. Am J Biomed Sci \& Res. 2019 - 4(1). AJBSR.MS.ID.000747. DOI: 10.34297/AJBSR.2019.04.000747

Received: July 09, 2019 | Published: July 15, 2019

\begin{abstract}
Modern Internal Medicine is faced with the need to ensure continuity of care by operating in highly technological contexts. The complexity in managing today's health needs must adapt to the growing articulation of demand and, on this basis, create flexible responses to emerging opportunities. Nowadays we are witnessing a new evolution in communication, with the expansion of virtual technologies that increasingly replace traditional forms. In this article we will briefly discuss the feasibility and the impact of new medical technologies, communications set and devices application in Internal Medicine, with particular attention to chronic patients, with prolonged interventions to support a growing fragility, favouring integration in health, social and welfare services..
\end{abstract}

Keywords: Information and Communication Technologies (ICT); Chronic Diseases; Telemedicine; Health Services

\section{Introduction}

Modern Internal Medicine is faced with the need to ensure continuity in care and, simultaneously, to operate in hightech and highly skilled contexts. The complex nature of today's health has to adapt to the growing articulation of demand and, on this basis, create flexible responses to emerging opportunities.

Plato, in the Phaedrus (275d), through the words of Socrates, introduces the question about communication and the technologies that convey it: "The writing, or Phaedrus, has this characteristic in a terrible way ... and so do the speeches (put in writing): you might believe that they speak as if they had some thoughts, but if you want to learn and question a speech about something that it says, it shows you one and the same thing. Once it is put in writing, every speech circulates everywhere, between the competent and equally between those who do not care and does not know who to turn and who does not."

The contrast between oral communication and written reflects an historical phase of transition in the Greek culture, in which the medium of writing was supplanting the normal channels of communication, changing the global communication system [1].

Nowadays we are witnessing a new evolution in communication [2-6], with the expansion of virtual technologies that increasingly replace traditional forms. This passage cannot fail to have methodological implications in health organization and in clinical practice, including the relationship between doctor and patient [7].

Although there are many concerns about possible degeneration, especially in the social relational field, related to new technologies [7], we are aware of the ineluctability of this process. Hence the need for a pragmatic approach, no longer asking whether ICT is useful in medical practice but rather how to grasp its positive aspects in clinical organization redesign.

Towards the end of the 1990s, healthcare companies began to analyze production processes. It was understood that the simple control of input factors in the black box representing the healthcare company is not sufficient to guarantee the achievement of quality, safety and efficiency targets in the provision of health services. A subsequent area of work was represented by the improvement of the hospital information systems attending to resolve problems of clinical relevance. In the meantime, there has been a tremendous progress in medicine as well as in informatics. One important benefit of this progress is that our life expectancy is nowadays significantly higher than it would have been even some few decades ago. This progress, leading to aging societies, influence health care organization and the development of its information systems [8]. 
Among recurrent research themes in healthcare setting dominated those related to the choice of the model's management, related to the introduction of new technologies that may redesign the daily clinical approach as well as patient-physician relationships [9]. Use of telemedicine is increasingly becoming an alternative therapeutic choice, especially in chronic diseases management, progressively rising all over the world, as well as the costs for their management. Health care expenditures and hospitalization experiences are to be considered among elderly populations with multiple chronic conditions. High-cost patients are more frequent in the sickest and most complex populations and their high resources utilization is primarily explained by high levels of chronic illness [10]. Moreover, patients with four or more chronic conditions were 99 times more likely than a patient affected by single disease to have an admission for an ambulatory care sensitive condition; in addition, per capita expenditures increased dramatically with 4 or more types of chronic conditions [11].

Information and communication technologies (ICT) are essential to optimize the results of the Chronic Care Model (CCM), for the prevention and management of chronic diseases. At the same time, the CCM can offer a methodological basis to improve the appropriateness of the use of ICT in health care. The two aspects complement each other to exploit each other's potential. However, in literature, there seem to be few points of contact between the thematic fields of "health service development" and "ICT innovation" [12]. It is necessary to fill these gaps by multiplying studies in this field, as the synergies between these sectors have enormous potential. Researchers, health policy makers and funding agencies must facilitate the pooling of forces between ICT experts and the redesign of the chronic care system, to effectively address the current epidemic of multiple long-term conditions.

To date health information technology literature demonstrates that implementing a multifunctional system can yield real benefits in terms of enhanced monitoring and surveillance activities, reduction of medication errors and decreased rates of utilization for potentially redundant or inappropriate care. However main experiences were designed by academic research institutions [13] and the effectiveness of these technologies in practice settings where most health care is delivered remains less clear.

From the point of view of reducing health costs, optimizing resources and improving access to health services, has to be considered the development of more complex telemedicine projects. These programs range from remote diagnostic services (for example in radiology through the transmission of data produced by diagnostic tools, with the possibility of having a second clinical evaluation on a patient) to clinical-medical apps, which allow the user to manage one's health through one's mobile phone or educating in disease's prevention, and services of remote visits through video calls.

An example is the initiative promoted by the Italian Diabetes Foundation for a tele-counseling and tele-education service for patients of all ages suffering from diabetes mellitus 1 . The project, started as a clinical study and carried out by our Medicine Department and Niguarda Hospital in Milan, allowed to compare a service of diabetological, dietological and psychological teleconsultations, performed via Skype ${ }^{\circ}$, with that of usual outpatient visits, demonstrating same effectiveness of the two approaches, allowing the patients followed in tele-consulting a significant saving in terms of time and costs (in particular those related to absences from work or school). Patients proved to be very satisfied, without having the perception of poor interaction with the doctor [14].

Together with telemedicine experience we designed an ongoing project to evaluate the accuracy in daily clinical use of a novel non-invasive fingertip-mounted device, designed to measure a wide range of physiological bio-parameters without the need to take painful capillary or venous invasive blood samples, in a large population of patients with peculiar characteristics, in particular clinical and assistance complexity and poly-morbidity.

These elements must also be considered from the point of view of the patients and the social nucleus to which they belong, the organization of the social-health services system and the relative system policies. A correct action of each of the components allows to tend towards the appropriateness and sustainability of the demand and the response, both in terms of service (usability, competence, effectiveness, efficiency) and of economic support (allocation of resources, verification of the correct allocation, resource allocation forecast).

For these reasons, in our clinical context, we design different projects to evaluate feasibility and organizing impact of new medical technologies, communications sets and devices in Internal Medicine wards, with particular attention to chronic patients and continuity of assistance, linking the cure of the acute phase of the disease to reintegration in daily life environment, with prolonged interventions to support a growing fragility, favouring integration in health, social and welfare services.

One of these projects is integration of tele-monitoring technologies in the protected discharge service, which allows the connection between hospital and territory, to improve the quality of care and affect clinical outcomes. Patients enrolled were selected between those discharged from Internal Medicine ward and with complex characteristics (such as a physical or mental pathology with a high severity index, not autonomous in the various activities, critical issues related to housing, income, family unit and so on).

Tele-monitoring system consists of a suite of commercial electro-medical instruments and a Smartphone with an Android operating system dedicated software, providing reminders at the time of scheduled surveys, transmitting data to a listening centre, where parameters detected with the available equipment (pulse oximeter, electronic sphygmomanometer, electronic scale and electrocardiograph) were collected. During the study period data were monitored by a dedicated multi-professional staff (nurse, GP, Internist) that may alert the patient in event of failure to transmit parameters, in the presence of acutely out-of-range values or detection of a unfavourable trend. Following the evaluation by the attending physician, the subsequent individual diagnostic and therapeutic plan will be planned and shared with an Internist, with a constant patients and care-giver involvement and empowerment. 
Our experience in remote patient monitoring taught us that, in terms of appropriateness, it is essential to monitories unstable patients, who have undergone several hospitalizations and who must be monitored immediately after discharge. The first month after discharge, when we performed the majority of therapeutic adjustments, is the most critical period in which monitoring is important to avoid re-hospitalization.

Tailored ICT application in the real world of Internal Medicine wards, to meet the needs of high-cost patients and to avoid waste of scarce resources, confirmed their favourable application. We believe that ICT may play a crucial role in chronic patients management as facilitate rationalization in human resources management and improve quality of care, making possible a more intense exchange between operators and between operators and patients, reducing the social cost of pathologies (in terms of time spent for users), and strengthening the patients and caregivers empowerment.

\section{References}

1. Vegeti M (1989) In the shadow of Theuth. Writing dynamics in Plato. In: M Detienne (Ed.), Knowledge and writing in Greece. Rome-Bari, pp. 201-227.

2. Stefani I, Mazzone A. Telemedicina, beautiful maignorata in Italy: that's why.

3. Lee J, Reyes BA, McManus DD, Mathias O, Chon KH (2012) Atrial fibrillation detection using a smart phone. Conf Proc IEEE Eng Med Biol Soc 2012: 1177-1180.

4. Nguyen HH, Silva JN (2016) Use of smartphone technology in cardiology. Trends Cardiovasc Med 26(4): 376-386.
5. Kaliyadan F, Amin TT, Kuruvilla J, Ali WH (2013) Mobile teledermatologypatient satisfaction, diagnostic and management concordance, and factors affecting patient refusal to participate in Saudi Arabia. J Telemed Telecare 19(6): 315-319.

6. Mazzone A (2015) iPhone ${ }^{\circledR}$ or smartphone support diagnosis in internal medicine. Italian J of Med 9 (2): 93.

7. Rosenthal DI, Verghese A (2016) Meaning and the nature of physicians work. N Engl J Med 375(19): 1813-1815.

8. Haux R (2006) Health information systems: past, present, future. Int J Med Inform 75(3-4): 268-281.

9. Stefani I, Teti O, Mazzone A (2017) Person-centred analysis in physicianpatient relationship. Eur J Intern Med 43: e26-e27.

10. Wammes JJG, van der Wees PJ, Tanke MAC, Gert P Westert, Patrick P T Jeurissen (2018) Systematic review of high-cost patients' characteristics and healthcare utilization. BMJ Open 8(9): e023113.

11. Wolff JL, Starfiel B, Anderson G (2002) Prevalence, expenditures, and complications of multiple chronic conditions in the elderly. Arch Intern Med 2002; 162(20): 2269-2276.

12. Gammon D, Berntsen GK, Koricho AT, Sygna K, Ruland C (2015) The chronic care model and technological research and innovation: a scoping review at the crossroads. J Med Internet Res 17(2): e25.

13. Chaudhry B, Wang J, Wu S, Maglione M, Mojica W, et al. (2006) Systematic review: impact of health information technology on quality, efficiency, and costs of medical care. Ann Intern Med 144(10): 742-752.

14. Bertuzzi F, Stefani I, Rivolta B, Pintaudi B, Meneghini E, et al. (2018) Teleconsultation in type 1 diabetes mellitus (TELEDIABE). Acta Diabetol 55(2): 185-192. 